\title{
Investigation of parameters of multimode four-layer waveguide structures and their influence on light propagation
}

\author{
Elżbieta Auguściuk, "Grzegorz Biniecki \\ Faculty of Physics, Warsaw University of Technology, Koszykowa 75, 00-662 Warsaw
}

Received July 21, 2009; accepted September 29, 2009; published September 30, 2009

\begin{abstract}
In the paper investigation of multimode four-layer waveguide structures by modified m-line spectroscopy method was presented. Waveguide layers with a gradient profile of the refractive index were prepared by the ion-exchange method. Dielectric layers were deposited on the waveguide by the spin-coating method. The investigation was aimed at learning the influence of parameters of the waveguide layer and the thin layer deposited on the waveguide on the propagation of light in this waveguide.
\end{abstract}

In miscellaneous experiments of integrated optics, thin dielectric layers are used as waveguides. Basic parameters of these layers are the refractive index $n_{f}$ and their thickness $W$. One of the methods to evaluate these waveguide parameters is the m-line spectroscopy method [1]. This method consists in light coupling into the waveguide using, for instance, a prism. Laser beam, which is coupled into the waveguide through the prism, drops on the base of the prism under the angle $\theta_{m}$.

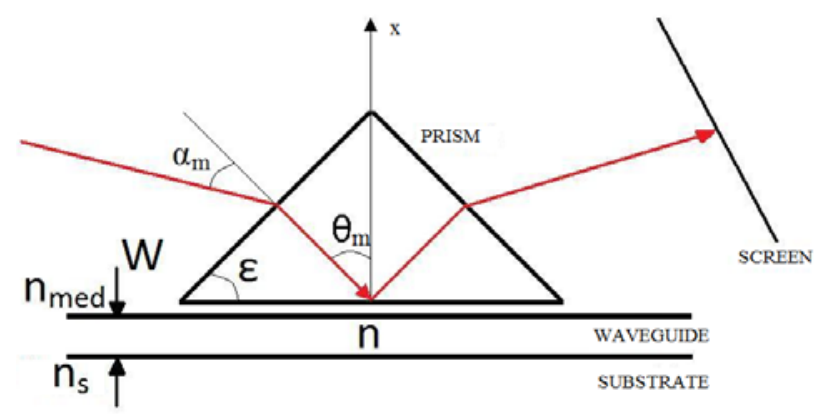

Fig.1. Schematic cross profile of a prismatic coupler to a waveguide

Experimental measuring arrangement, which was used to observe light coupling to a waveguide and to measure the coupling angles, is shown in Figures 1 and 2.

*E-mail: eaugust@if.pw.edu.pl

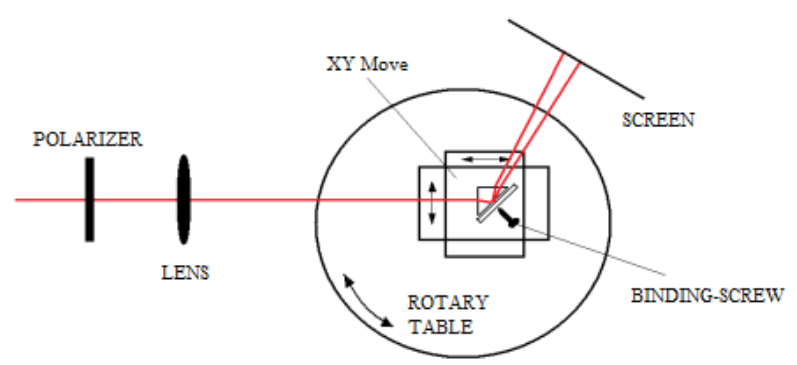

Fig.2. Schematic measurement setup

The waveguide with a prism occurs in a suitable holder in which the waveguide is pressed to the base of the prism by a screw. This handle with the waveguide and prism are on the platform, which can move in XY direction, and everything takes place on a rotary table. The laser beam has linear polarization (TE or TM). The lens converges the laser beam in the pressing point. The optical setup is regulated in such a way that the point of coupling light is stable, during the rotation of the table.

Theoretical analysis of the problem with propagation light in the waveguide shows dependence between the distribution of effective indices of the successive modes and the profile of a refractive index of this waveguide. The assumption is a monotonical decrease of refractive index $n(x)$ inwards the waveguide. Effective indices $N_{m}$ are evaluated by measuring the incident angles into the prism face $\alpha_{m}$ the moment light is coupled into the waveguide.

The refractive index profile $n(x)$ is calculated by resolving the dispersion equation (1) where known are $\alpha_{m}$ angles [2].

$$
\int_{0}^{x_{m}^{t}}\left(n^{2}(x)-N_{m}^{2}\right)^{\frac{1}{2}} d x=F_{m}
$$

where: 


$$
F_{m}=\frac{1}{k}\left\{m \pi+\frac{\pi}{4}+\tan ^{-1}\left\{\left[\frac{n(0)^{2}}{n_{m e d}^{2}}\right]^{\sigma} \cdot\left[\frac{\left(\zeta_{m}+n_{s}^{2}-n_{m e d}^{2}\right)}{\left(n(0)^{2}-\zeta_{m}-n_{s}^{2}\right)}\right]^{\frac{1}{2}}\right\}\right\}
$$

where $\zeta_{m}=N_{m}{ }^{2}-n_{s}{ }^{2}$

The solution of this dispersion equation is in progress with the assumption that the function $F_{m}\left(\zeta_{m}\right)$ has a polynomial form.

Effective propagation indices $N_{m}\left(\alpha_{m}\right)$ are approximated in the range $m=(-3 / 4 ; N)$ by an orthogonal polynomial. If approximated error $S$ is higher than experimental error $\Delta$ then the degree of polynomial is enlarged by one until the moment when $S \leq \Delta$. The function $N_{m}(m)$ is used to calculate $n(0)$ :

$$
n(0)=N_{m}\left(m_{i}=-0,75\right)
$$

In the same way function $F_{m}\left(\zeta_{m}\right)$ is approximated. Such a form of the function $F_{m}(\zeta)$ and $N_{m}\left(m_{i}\right)$ provides the integration of the equation (1), which makes it possible to calculate the refractive index profile $n(x)$.

$$
x(f)=-\frac{2}{\pi} \int_{f(0)}^{f} \frac{\frac{d F_{m}(\zeta)}{d \zeta} d \zeta}{\sqrt{\zeta-f}}
$$

where $f=n_{f}^{2}(x)-n_{s}^{2}$.

In the case when the waveguide is produced by ion exchange the method has a monotonically decreasing refractive index (Fig.3.). Dielectric layers are deposited on the waveguide by a spin-coating method and have a step-index profile of the refractive index. The presence of a thin cover layer on the waveguide influences its propagation characteristics. A generalized m-line spectroscopy method [2, 3] enables the evaluation of the parameters of the subguiding layers deposited on a waveguide.

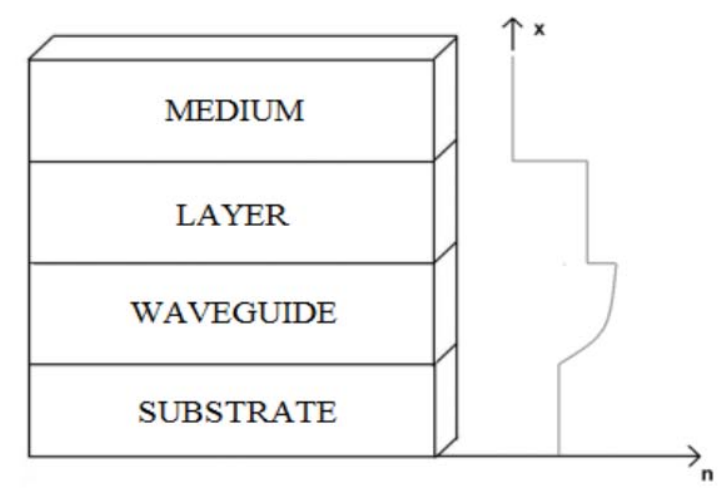

Fig.3. Waveguide with thin layer
Dispersion equation has the following form:

$$
\begin{aligned}
& b^{\prime} X_{m}= \\
& \tan ^{-1}\left(\frac{p_{s}}{b^{\prime}}\right)+\tan ^{-1}\left(\frac{p_{l}}{b^{\prime}} \cdot \frac{p_{\text {med }} \cosh \left(p_{l} t\right)+p_{l} \sinh \left(p_{l} t\right)}{p_{l} \cosh \left(p_{l} t\right)+p_{\text {med }} \sinh \left(p_{l} t\right)}\right)+m \pi
\end{aligned}
$$

where $p_{l}=\sqrt{n_{l}^{2}-\left(N_{m}^{\prime}\right)^{2}} ; p_{j}=\sqrt{\left(N_{m}^{\prime}\right)^{2}-n_{j}^{2}} ; \mathrm{j}=\mathrm{s}$, med.

The left side of dispersion equation can be written in the following form:

$$
b^{\prime} X_{m}=k_{0} \int_{0}^{x_{m}} \sqrt{n^{2}(x)-\left(N_{m}^{\prime}\right)^{2}} d x
$$

SO

$$
b^{\prime}=\frac{k_{0} \int_{0}^{x_{m}} \sqrt{n^{2}(x)-\left(N_{m}^{\prime}\right)^{2}} d x}{X_{m}}
$$

where: $n(x)$ - waveguide refractive index profile, $\mathrm{N}_{\mathrm{m}}$-effective propagation indices for the waveguide with a thin layer

Using this equation we can get a depth of penetration and value of parameter $b$ '. Dispersion equation after easy transformations has the form [3]:

$$
t=\frac{1}{p_{l}} \operatorname{coth}^{-1}\left(\frac{\left(p_{l}^{2}-p_{m e d} \cdot b^{\prime} \cdot \tan C\right.}{p_{l}\left(b^{\prime} \cdot \tan C-p_{\text {med }}\right)}\right)
$$

where:

$$
C=b^{\prime} X_{m}-m \pi-\arctan \left(p_{s} / b^{\prime}\right)
$$

In equation's left side is represented by layer's thickness, although on the right side of equation there is only one unknown value - a layer refractive index, so we know the function $t\left(n_{l}\right)$. The function $\phi\left(n_{l}\right)$ is the difference between layer thickness for mode "a" and mode " $b$ ".

$$
\Phi\left(n_{l}\right)=t\left(n_{l}\right)_{a}-t\left(n_{l}\right)_{b}
$$

The function $\phi\left(n_{l}\right)$ owns zeros in points, which layer thickness for mode $a$ and mode $b$ is equal. To obtain the layer refractive index it is necessary only to find zero points. Then, layer thickness is calculated from equation (4).

After the measurement of all incident angles we calculate characteristic waveguide parameters, their thickness $W$, refractive index $n(0)$ and the refractive index profile. 
During the production of waveguides we could change parameters of ion exchange, that is the time and temperature of ion exchange. These changes when the time of ion exchange was constant $\left(t_{\text {wym }}=20 \mathrm{~min}\right.$; $t_{\text {wym }}=$ $15 \mathrm{~min}$ ) are presented in the Tables 1 and 2. We compare waveguides which were made by Gevert glass $(n=1,5045)$.

Table 1. Waveguide characteristic parameters, $t_{w y m}=20 \mathrm{~min}$

\begin{tabular}{|c|c|c|c|c|}
\hline $\begin{array}{c}\text { Waveguide } \\
\text { number }\end{array}$ & $\begin{array}{c}\boldsymbol{T}_{\text {stopu }} \\
{\left[{ }^{\circ} \boldsymbol{C}\right]}\end{array}$ & $\boldsymbol{W}[\boldsymbol{\mu m}]$ & $\boldsymbol{n}_{\boldsymbol{f}}$ & $\begin{array}{c}\text { Modes } \\
\text { quantity }\end{array}$ \\
\hline 1 & 380 & $8,9 \pm 0,2$ & $1,5944 \pm 0,030$ & 12 \\
\hline 2 & 378 & $8,4 \pm 0,2$ & $1,5877 \pm 0,030$ & 11 \\
\hline 3 & 372 & $7,5 \pm 0,2$ & $1,5758 \pm 0,030$ & 9 \\
\hline 4 & 369 & $6,5 \pm 0,2$ & $1,5599 \pm 0,030$ & 7 \\
\hline 5 & 365 & $6,1 \pm 0,2$ & $1,5559 \pm 0,030$ & 6 \\
\hline
\end{tabular}

For smaller time of ion exchange $t_{w y m}=15$ min waveguide characteristic parameters are shown in table 2.

Table 2. Waveguide characteristic parameters, $t_{\text {wym }}=15 \mathrm{~min}$

\begin{tabular}{|c|c|c|c|c|}
\hline $\begin{array}{c}\text { Waveguide } \\
\text { number }\end{array}$ & $\begin{array}{c}\boldsymbol{T}_{\text {stopu }} \\
{\left[{ }^{\circ} \mathrm{C}\right]}\end{array}$ & $\boldsymbol{W}[\boldsymbol{\mu m}]$ & $\boldsymbol{n}_{\boldsymbol{f}}$ & $\begin{array}{c}\text { Modes } \\
\text { quantity }\end{array}$ \\
\hline 6 & 385 & $8,9 \pm 0,2$ & $1,5945 \pm 0,030$ & 12 \\
\hline 7 & 383 & $8,6 \pm 0,2$ & $1,5899 \pm 0,030$ & 11 \\
\hline 8 & 380 & $8,2 \pm 0,2$ & $1,583 \pm 0,030$ & 10 \\
\hline 9 & 372 & $6,7 \pm 0,2$ & $1,5618 \pm 0,030$ & 7 \\
\hline
\end{tabular}

The next step was the calculation of characteristic parameters of layers which were deposited on waveguides. After measuring incident angles, we used the WAVEGUIDE program which helps us to calculate the parameters of thin layers, i.e. their thickness $t$ and refractive index $n_{l}$.

On waveguides two kinds of layers were deposited:

a) polystyrene

Table 3. Characteristic parameters of polystyrene layers

\begin{tabular}{|c|c|c|}
\hline Number & $\boldsymbol{t}[\boldsymbol{\mu m}]$ & $\boldsymbol{n}_{\boldsymbol{l}}$ \\
\hline $\mathbf{6}$ & $1,089 \pm 0,034$ & $1,6485 \pm 0,0036$ \\
\hline $\mathbf{7}$ & $1,084 \pm 0,034$ & $1,6453 \pm 0,0034$ \\
\hline $\mathbf{1 6}$ & $1,074 \pm 0,034$ & $1,6458 \pm 0,0037$ \\
\hline $\mathbf{1 7}$ & $1,088 \pm 0,034$ & $1,6459 \pm 0,0036$ \\
\hline
\end{tabular}

b) poly(vinyl acetate)
Table 4. Characteristic parameters of poly(vinyl acetate) layers

\begin{tabular}{|c|c|c|}
\hline Number & $\boldsymbol{t}[\boldsymbol{\mu m}]$ & $\boldsymbol{n}_{\boldsymbol{l}}$ \\
\hline $\mathbf{1}$ & $1,051 \pm 0,039$ & $1,6374 \pm 0,0033$ \\
\hline $\mathbf{1 0}$ & $1,055 \pm 0,055$ & $1,6385 \pm 0,0041$ \\
\hline $\mathbf{2}$ & $1,050 \pm 0,039$ & $1,6354 \pm 0,0032$ \\
\hline
\end{tabular}

During the process of depositing the layers on waveguides the first thing which we did was making a choice of proper substances. In our case it was polystyrene and poly(vinyl acetate). One of the chosen substances has the refractive index higher than the refractive index of waveguide; the second one has a lower refractive index. The layer thickness was equal to about $1 \mu \mathrm{m}$.

However, polystyrene layers in all cases have a larger thickness than poly(vinyl acetate) layers. It was caused by the fact that polystyrene has a higher density and so a higher refractive index than poly(vinyl acetate).

Refractive layer's indices which we calculated are different from bulk values. It is caused by different atom's positions in thin layers than in bulk substance, so thin layers have different properties and it is the reason why refractive indices are different from bulk values. However, in our experiments a difference between a polystyrene refractive index and a poly(vinyl acetate) refractive index was kept, but both layers had a refractive index larger than a waveguide refractive index.

\section{References}

[1] R. Ulrich R. Torge, „Measurement of Thin Film Parameters with a prism coupler,” Applied Optics 12, 2901 (1973).

[2] E. Auguściuk, F. Sala, „Application of planar waveguide with gradient index profile to determine parameters of thin active layers used in waveguide sensor”, Proc. of SPIE vol. 6585, D1 (2007)

[3] E. Auguściuk, „Multilayer waveguide structures investigated by the generalized m-line spectroscopy”, Proc. SPIE vol. 7120, 71200J (2008). 